\title{
Imaging of Hematological Patients in the Era of COVID-19
}

\author{
Yael Eshet ${ }^{\mathrm{a}, \mathrm{b}} \quad$ Abraham Avigdor $^{\mathrm{b}, \mathrm{c}}$ Meirav Kedmi ${ }^{\mathrm{b}, \mathrm{c}}$ Noam Tau $^{\mathrm{b}, \mathrm{d}}$ \\ aDepartment of Nuclear Medicine, Sheba Medical Center, Ramat Gan, Israel; 'backler School of Medicine, \\ Tel Aviv University, Tel Aviv, Israel; ' Hematology Division, Chaim Sheba Medical Center, Tel Hashomer, Israel; \\ ${ }^{\mathrm{d}}$ Department of Diagnostic Imaging, Sheba Medical Center, Ramat Gan, Israel
}

\section{Keywords}

Hematology · COVID-19 · Radiology · Positron emission tomography-computed tomography · Nuclear medicine

\begin{abstract}
The COVID-19 pandemic, caused by the severe acute respiratory syndrome coronavirus 2 (SARS-CoV-2), resulted in changes in management and imaging routines for patients with hematological malignancies. Treating physicians had to familiarize themselves with a new disease, with distinct imaging manifestations, sometimes overlapping with other infections prevalent in this patient population. In some aspects, infected hematological patients might exhibit a different disease course, and routine imaging in asymptomatic hematological patients may result in unexpected COVID-19 findings, implying covert infection, that should be further explored. Furthermore, some complications of hematological diseases and treatments may present with findings similar to COVID-19 manifestations, and treating physicians must consider both possibilities in the differential diagnosis. In this review, we aimed to present the influence the COVID-19 pandemic had on hematological malignancy imaging.

(c) 2022 S. Karger AG, Basel
\end{abstract}

\section{Introduction}

COVID-19, which was declared a world pandemic by the World Health Organization in the beginning of March 2020, has transformed the lives of millions of people, with repercussions on patients and their caretakers. For patients with hematological diseases, lockdown changed management and imaging routines. Fear of contracting the disease and lockdown orders discouraged some people from unnecessary medical encounters, at least at the initial stages $[1,2]$. Caretakers had to familiarize themselves with the characteristics of a new disease, and differential diagnosis for symptoms and imaging findings had to be expanded to include COVID-19 manifestations.

This review will describe common imaging findings of COVID-19 infection (mainly partaining to the alfa and delta variants), clinical COVID-19 presentations specific to hematological patients, and COVID-19-related imaging findings, which mimic typical findings and complications in hematological patients. We also present typical imaging findings after mRNA-based COVID-19 vaccination, which may mimic findings usually more typical for hematological malignancies.

Correspondence to:

Yael Eshet, yael.eshet@gmail.com 


\section{Main Imaging Findings in COVID-19 Pneumonia}

The majority of COVID-19 manifestations are related to the respiratory tract. Chest X-ray could be a triage tool in symptomatic patients [3] with suspected COVID-19 infection, showing multilobular, pulmonary groundglass opacities (GGOs), and consolidations, especially in bilateral and peripheral lower lobe distribution, consistent with pneumonia [4]. However, these findings are in no way specific for COVID-19, and there is an overlap with other diseases, some common in hematological patients, including other viral infections (including influenza and others); bacterial infections (including atypical pathogens, such as Mycoplasma pneumoniae); fungal infections (mainly Pneumocystis jiroveci [PJP]); and other noninfectious diseases [5-7]. In most hospitals, dedicated chest X-ray rooms were used to image suspected COVID-19 patients, with protection measures for the attending stuff. Although it is a powerful, cheap, and available imaging modality, about $30 \%$ of COVID- 19 patients requiring admission and $20 \%$ of hospitalized patients had normal chest X-rays [8].

Chest computed tomography (CT) is more sensitive in detecting the typical COVID-19 pulmonary findings, especially GGOs and reticular infiltrates. Although chest CT was found to be positive in some patients with negative real-time reverse transcription-polymerase chain reaction (RT-PCR), it is not advised as a first-line screening tool in the diagnosis of COVID-19, but reserved for assessment of complications in symptomatic, hospitalized patients, such as patients with suspected pulmonary embolism [3].

Chest CT findings are typically chronologically related to symptom's onset. These include small, subpleural, unilateral, or bilateral GGOs in the lower lobes (peaking on days 12-17 from the patient's symptom onset), turning into a "crazy paving" pattern, which eventually transforms into pulmonary consolidations. These findings typically subside, leaving subpleural parenchymal bands, and finally resolve, usually within $2-3$ weeks $[9,10]$.

\section{Protracted COVID-19 Pneumonia in B Cell-Depleted Patients}

B cell-depleted patients may be protected from COVID-19-associated cytokine storm. However, they may have difficulties in viral clearance leading to a protracted pneumonia [11, 12]. Some COVID-19 patients receiving anti-CD20 treatment were described as showing a pro- longed course of COVID-19-associated recurring-remitting pneumonia, apparent also on chest imaging 3 [11], 6 [13], and even 12 months [14] after initial COVID-19 symptoms. This was not described in immunocompetent patients, as the acute phase of COVID-19 usually subsides within a few days or weeks after symptoms begin $[15,16]$. In our hospital, we encountered a case of a 78-year-old patient, who contracted COVID-19 infection 4 months after cessation of bendamustine-rituximab treatment for splenic marginal zone lymphoma, with repeat hospitalizations due to relapsing, migrating pneumonia, confirmed by CT and positron emission tomography (PET)CT scans (shown in Fig. 1), lasting 7 months after initial COVID-19 diagnosis.

\section{Challenges in Imaging Patients with Hematological Malignancies during the COVID-19 Pandemic}

Patients with hematological malignancies are frequent visitors to the radiology and nuclear medicine departments, as most patients with aggressive lymphomas are staged, evaluated for response, and followed up by PETCT [17], with frequent visits, as often as every 2 to 3 months during active treatment. Indolent lymphoma patients are usually evaluated by CT, but can also be referred to PET-CT when transformation to aggressive disease is suspected. Some patients with multiple myeloma are staged or followed up by CT, magnetic resonance imaging (MRI), or PET-CT, as well as some leukemia patients assessed for extramedullary diseases [18]. Moreover, these patients can be referred for imaging when complications are suspected. Since these visits are deemed critical for patient management, they were not rescheduled during COVID-19 outbreaks. As a result, some asymptomatic patients were diagnosed with COVID-19 following routine imaging.

\section{PET-CT Unit Performance during Lockdown}

Most guidelines for performing PET-CT during the pandemic stated that 18-fluorodeoxyglucose (FDG) PETCT for staging or therapy response assessment should not be postponed in patients without known active COVID-19 infection [19]. In fact, the workload in nuclear medicine departments was less affected by the initial worldwide lockdown (between March and May 2020) than other imaging modalities, with rebound resurgence in scan numbers in the following months [20]. A study conducted in 7 US medical centers found that PET-CT specifically was consistently the least impacted imaging 

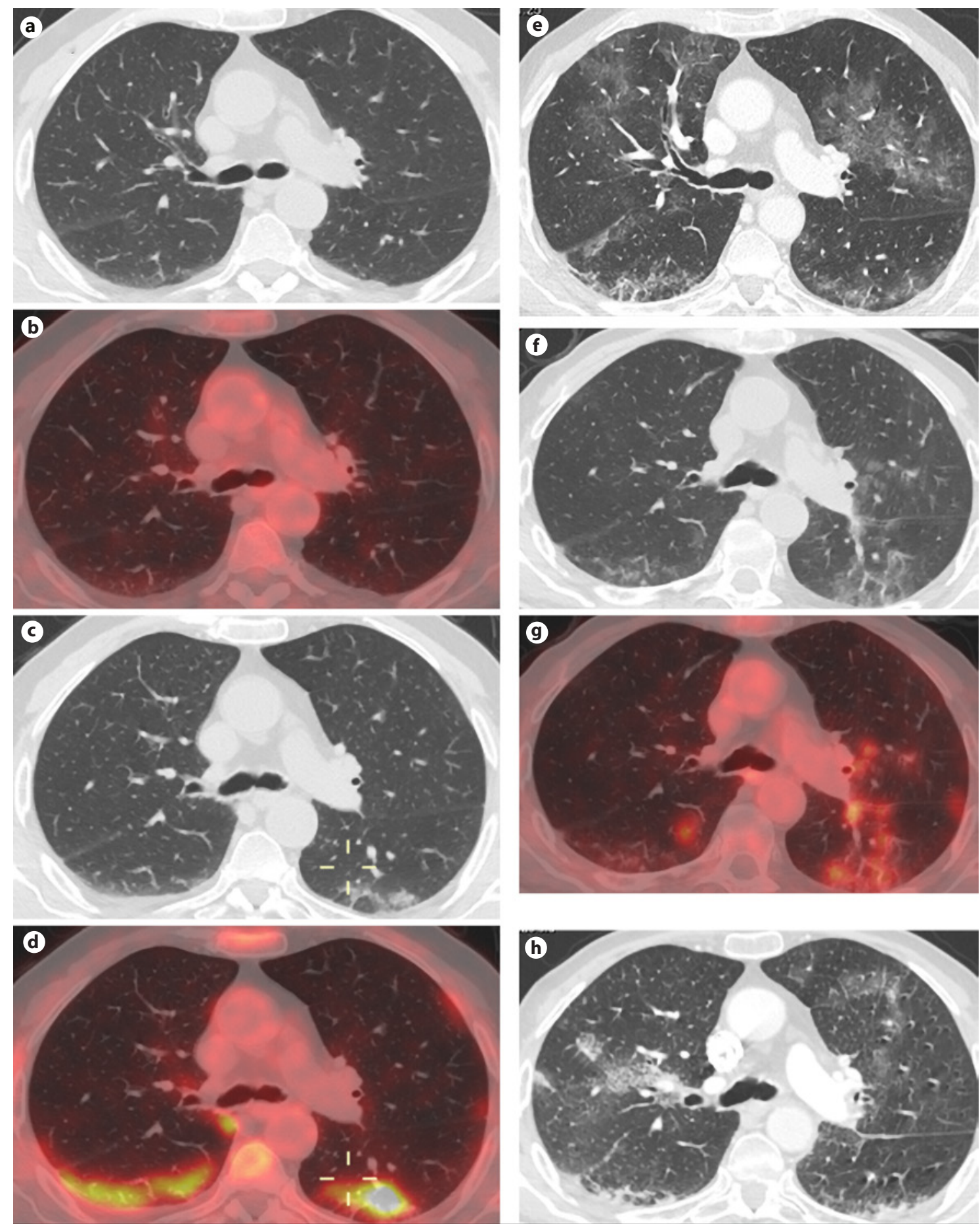

Fig. 1. A 78-year-old patient, who contracted COVID-19 4 months after BR treatment cessation for follicular lymphoma. Relapsing remitting episodes of fever and pneumonia, tests negative for other pathogens. $\mathbf{a}, \mathbf{b}$ Before COVID-19 infection. No pulmonary findings. c, d One week after symptoms onset. High FDG uptake in lower lobes peripheral pulmonary infiltrates. e One month after primary COVID infection. Recurring, migrating GGOs. f, $\mathbf{g}$ Three months after primary infection. Mild FDG uptake in peripheral lower lobes reticular infiltrates. h Seven months after primary COVID infection. New GGOs in upper lobes and subpleural reticular infiltrates in lower lobes. BR, bendamustin-rituximab. 
Fig. 2. Matched FDG-PET-CT scan number between lockdown and comparable period the prior year in a single tertiary hospital. While a small drop in scan numbers was noted in the first two lockdown months in 2020 , there was a rebound effect in the following months.

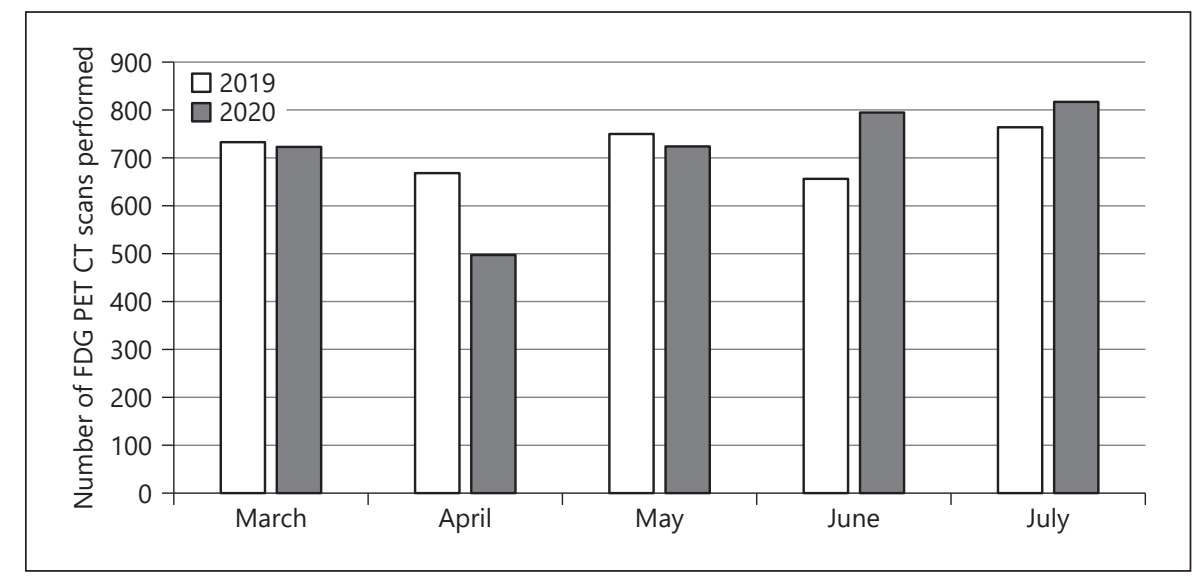

modality, with a drop of $16 \%$ in patient output, more pronounced in centers located at pandemic outbreak locations [21]. In our tertiary hospital, there was no change in number of FDG-PET-CT performed at the beginning of lockdown in March 2020, a minimal reduction in April compared to the previous year, and a later rebound in June and July 2020 (shown in Fig. 2). There was no effective change in annual scan numbers.

\section{Incidental COVID-19 Diagnosis in Asymptomatic}

Patients Performing PET-CT

PET-CT in not the optimal first-line imaging modality used in COVID-19 patients, due to its high radiation exposure, lower availability, long acquisition time (raising the risk of disease spread) [22], and high cost. Unlike Xray or CT suites, which could be dedicated to biohazard patients, most facilities cannot afford to dedicate a PETCT scanner solely for COVID-19 patients, and the room and equipment must be decontaminated after scanning a known COVID-19 patient [23, 24].

However, asymptomatic patients with hematological malignancies undergoing frequent and routine PET-CT, may exhibit pulmonary findings consistent with previously unknown COVID-19 infection. Such findings can also be spotted in known, convalescing patients, usually lasting up to 2 weeks after symptoms onset. These findings usually include characteristic multiple peripheral GGOs with high uptake of the radioactive glucose analogue FDG [25], portraying anaerobic glycolysis taking place in neutrophils [26]. Higher maximal standard uptake values were noted at earlier stages of COVID-19 pneumonia infiltrates [27], with complete resolution of pulmonary findings and uptake within 4 weeks of symptom onset [28].
Reactive mediastinal and supraclavicular lymph node uptake was frequently detected in active COVID-19 patients [25, 28-32], occurring in normal-sized lymph nodes, which may therefore be left unnoted on regular chest CT scans [33]. Other nonpulmonary COVID-19 FDG-PET-CT findings include reduced metabolic activity in the frontal cortex of the brain (consistent with anosmia symptoms) [34] and increase in FDG uptake in the parotid/salivary glands [35].

\section{COVID-19 Imaging Mimickers of Common Hematological Findings}

Some imaging findings in active COVID-19 patients, convalescing COVID-19 patients, and subjects receiving anti-COVID-19 vaccinations, resemble imaging findings usually encountered in hematological patients. Recognizing them in these patients with known COVID-19 or in times of outbreak could broaden the differential diagnosis and reduce imaging misinterpretation.

As the sensitivity of chest CT may be greater than that of RT-PCR in diagnosing early COVID-19 (98\% vs. 71\%, respectively) [36], a suspicious CT scan with negative RTPCR does not exclude COVID-19 infection, rendering the differentiation more difficult. It should be noted that COVID-19 pneumonia often has the same features on chest CT in immunosuppressed and nonimmunosuppressed patients [37].

\section{Drug Toxicity Associated Pneumonitis versus COVID-19 Pneumonia}

Bilateral, multifocal, peripheral, and patchy GGOs, with mild FDG uptake, which are typical pulmonary find- 
ings in COVID-19 patients, are also typical findings in pulmonary drug toxicities, and the differentiation is difficult in hematological patients receiving chemotherapy or anti-CD20 medications in times of COVID-19 outbreaks. Karasu et al. [38] described 2 patients with drugrelated pulmonary infiltrates resembling COVID-19 pneumonia, with resolution of findings after drug withdrawal and steroid treatment. Dai et al. [39] described a patient with dyspnea and pneumonitis while treated with anti-PD-1 therapy, with persistently negative RT-PCR and good response to steroids and antibiotics.

However, patients presenting with fever, conjunctivitis, or gastrointestinal symptoms accompanying the pulmonary infiltrates are more likely to suffer from COVID-19 and not drug toxicity [40]. Headaches and sore throat, which are typical in COVID-19 infection, are possibly additional partitioning symptoms.

\section{Atypical Pneumonia versus COVID-19 Pneumonia}

COVID-19-related lymphopenia, prolonged intensive care hospitalization, and steroid treatment can render COVID-19 patients more prone to atypical, opportunistic infections, which are usually commonly seen only in immunocompromised patient.

Differentiating between pulmonary findings in COVID-19 and other entities common in hemato-oncological patients, such as aspiration pneumonia, tuberculosis, nocardiosis, and pulmonary Langerhans cell histiocytosis is beyond the scope of this review, but has been discussed elsewhere [41]. Interestingly, Giannakis et al. [42] found that thoracic radiologists were able to differentiate COVID-19 pneumonia from atypical pneumonias (influenza virus, parainfluenza virus, respiratory syncytial virus, cytomegalovirus, herpes simplex virus type 1, M. pneumoniae, Legionella pneumophila, and PJP) on chest CT scans with high overall accuracy (88\%) and specificity (90\%), but somewhat lower sensitivity (79\%), especially in the non GGOs stages (probably because GGOs are also commonly encountered in PJP, herpes simplex virus type 1 , and cytomegalovirus).

\section{Increased Bone Marrow and Spleen Uptake}

A pitfall in active COVID-19 infection includes diffusely increased bone marrow and spleen FDG uptake, which could mimic or obscure bone marrow involvement in hematological diseases [28, 32]. Interestingly, the spleen was also found to increase in size in the week following COVID-19 onset. Tahtbasi et al. [43] compared splenic indexes and splenic volume at COVID-19 infection presentation and in the following week, in 160 clini- cally deteriorating COVID-19 patients and found increase in spleen size, correlating with COVID-19 pneumonia severity. Both these findings may overlap disease relapse in patients with hematological malignancies and should be taken into account in the presence of PET-CT scan during active COVID-19.

Several case reports described splenic infarction incidentally detected on CT of nonhematological COVID-19 patients, probably related to their prothrombotic state [44-46]. In 1 study, 3 out of 209 critical care COVID-19 patients exhibited splenic infarct on imaging [47]. However, the incidence of this finding in noncritical care patients is unknown. As hematological patients with splenomegaly, splenic involvement, sickle cell traits, [48] or prothrombotic states are prone to splenic infarctions, this pitfall should also be recognized.

\section{Other COVID-19 Complications Mimicking}

Hematological Complications

COVID-19-associated mucormycosis $[49,50]$ was also described as a rare complication of critically ill COVID-19 patients, especially in India. Maini et al. [51] reported a case of mucormycosis established by MRI in a recovering COVID-19 patient with chemosis and left ocular pain. In addition, COVID-19-associated pulmonary aspergillosis has been described in an incidence ranging between 3 and 20\% among critically ill COVID-19 patients, mainly immunocompetent [52]. Of course, similar infections are seen in patients with hematological malignancies, and such an overlap should be taken into account when encountering these patients. Typhlitis, a life-threatening necrotizing enterocolitis usually described in neutropenic patient, was also described in a patient without prior hematological malignancy with COVID-19-associated pancytopenia [53].

\section{Vaccination-Associated Lymphadenopathy}

With the widespread emergence of the novel mRNA vaccinations, multiple vaccinated subjects presented with enlarged and sometimes painful lymph nodes in the vaccinated arm draining basin [54], which posed a diagnostic dilemma, especially in woman with known or suspected breast cancer, in melanoma patients of the thorax, head, and neck or arms, and in hemato-oncological diseases involving lymph nodes. Although FDG avid lymphadenopathy secondary to vaccinations has been described in $5-29 \%$ of patients after influenza vaccination $[55,56]$, it usually exhibited only mild FDG uptake and lasted for no more than 14-49 days [56, 57].

In the case of mRNA vaccinations against COVID-19, these seem to trigger a more extensive regional immune 


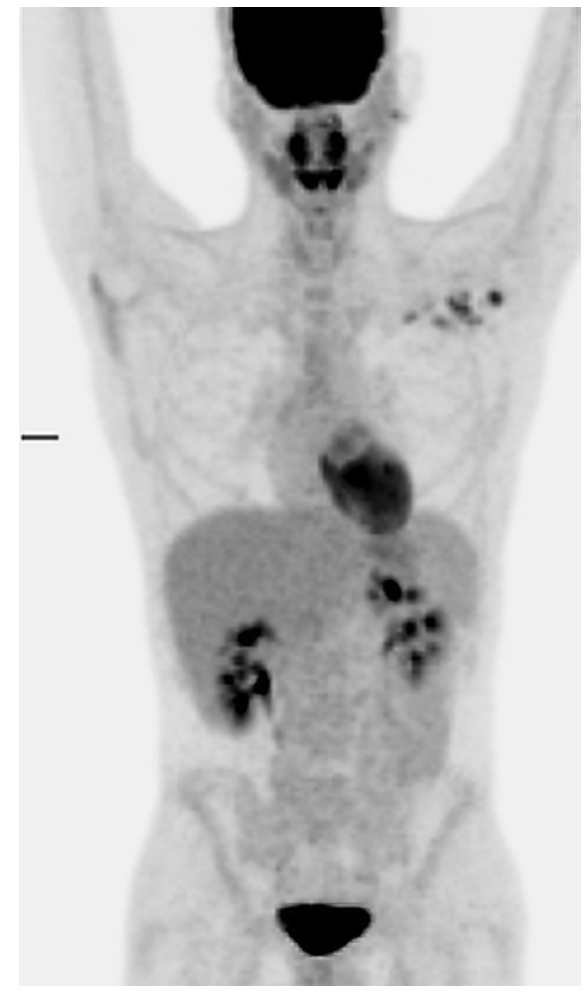

Fig. 3. A 21-year-old patient, follow-up scan 2 years after treatment for follicular lymphoma. Scan performed 4 days after left arm mRNA vaccination for COVID-19, showing avid left axillary lymphadenopathy. Under observation, the lymphadenopathy resolved.

response than reported for the influenza vaccine. Among vaccinated people, $14-66 \%$ of patients undergoing FDGPET-CT after anti-COVID-19 vaccination had avid axillary lymphadenopathy ipsilateral to the injection site [5861], as shown in Figure 3, with higher FDG uptake, and a prolonged response, reported to last even 10 weeks after the injection [62].

Knowledge of recent vaccination history can limit the rate of misdiagnosing these lymph nodes as pathological. Clinical and imaging follow-up will show resolution of lymphadenopathy. In highly controversial cases, a biopsy can be performed. Of note, immunosuppressed and hematological patients were significantly less prone to increased axillary lymph node uptake than the rest of the population [60].

\section{Vaccine-Induced Immune Thrombotic}

Thrombocytopenia

A rare complication of COVID-19 vaccines, namely those applying the adenovirus-vector technique (e.g., As-
traZeneca's and Johnson \& Johnson's COVID-19 vaccines), is vaccine-induced thrombotic thrombocytopenia (VITT), which was identified within 6 weeks of the vaccines' initial rollout [63]. VITT is characterized by thrombotic events occurring in various sites, including cerebral sinus vein thrombosis [64], pulmonary embolism, leg deep-vein thrombosis, and other less common sites (e.g., portal vein, splenic vein, and others). These usually present within 5-30 days after being vaccinated with an adenoviral-vectored COVID-19 vaccine and are more common in younger and female vaccine recipients. VITT's pathophysiology is not yet completely clear but has been suggested to be related to antigenic complexed formed between vaccine components and platelet factor 4 , therefore creating an anti-platelet factor 4 response [65]. Laboratory findings in classic VITT can include thrombocytopenia, elevated D-dimer levels, and decreased fibrinogen levels. When a thrombotic event is suspected in a patient who was recently vaccinated with one of the culprit vaccines, urgent targeted imaging is advised. Imaging options include compression ultrasound for suspected deep-vein thrombosis, pulmonary artery CT for suspected pulmonary embolism, and CT or MRI venography for any other site of suspected thrombosis.

\section{Conclusion}

The COVID-19 pandemic poses new challenges for patients with hematological malignancies and their treating physicians. With gained experience, having overcome the initial pandemic waves, we should be familiar with the typical imaging findings of COVID-19, diagnose previously unsuspected COVID-19 infections, recognize typical incidental imaging findings and acknowledge that some of the complications, previously more specific to hematological patients, can also represent COVID-19 (or COVID-19 vaccination) manifestations.

\section{Acknowledgments}

Dr. Elena Ribakovsky collected medical cases. Dr. Vered Dolberg suggested topics for the review.

\section{Statement of Ethics}

The patient in Figure 1 was part of a study reviewed and approved by the Sheba Medical Center Institutional Review Board, approval number 8176-21-SMC, informed consent was waived. 
The patient in Figure 3 was part of a study reviewed and approved by the Sheba Medical Center Institutional Review Board, approval number 8802-21-SMC, informed consent was waived.

\section{Conflict of Interest Statement}

The authors have no conflicts of interest to declare.

\section{Funding Sources}

No funding was received for this review.

\section{Author Contributions}

Conception and design: Y.E. and N.T. Clinical data collection: Y.E. and A.A. Script preparation and revisions: Y.E., N.T., M.K., and A.A.

\section{References}

1 Weinkove R, McQuilten ZK, Adler J, Agar MR, Blyth E, Cheng AC, et al. Managing haematology and oncology patients during the COVID-19 pandemic: interim consensus guidance. Med J Aust. 2020 Jun;212: 481-9.

2 Lai AG, Pasea L, Banerjee A, Hall G, Denaxas $\mathrm{S}$, Chang WH, et al. Estimated impact of the COVID-19 pandemic on cancer services and excess 1-year mortality in people with cancer and multimorbidity: near real-time data on cancer care, cancer deaths and a populationbased cohort study. BMJ Open. 2020 Nov 17; 10:e 043828

3 Rubin GD, Ryerson CJ, Haramati LB, Sverzellati N, Kanne JP, Raoof S, et al. The role of chest imaging in patient management during the COVID-19 pandemic: a multinational consensus statement from the Fleischner Society. Radiology. 2020 Jul;296:172-80.

4 Revzin MV, Raza S, Warshawsky R, D'Agostino C, Srivastava NC, Bader AS, et al. Multisystem imaging manifestations of COVID-19, part 1: viral pathogenesis and pulmonary and vascular system complications. Radiographics. 2020 Oct;40:1574-99.

5 Hochhegger B, Zanon M, Altmayer S, Mandelli NS, Stüker G, Mohammed TL, et al. COVID-19 mimics on chest CT: a pictorial review and radiologic guide. Br J Radiol. 2021 Feb 1;94:20200703.

6 Hanfi SH, Lalani TK, Saghir A, McIntosh LJ, Lo HS, Kotecha HM. COVID-19 and its mimics: what the radiologist needs to know. J Thorac Imaging. 2021 Jan;36(1):W1-10.

7 Duzgun SA, Durhan G, Demirkazik FB, Akpinar MG, Ariyurek OM. COVID-19 pneumonia: the great radiological mimicker. Insights Imaging. 2020 Nov 23;11:118.

8 Wong HYF, Lam HYS, Fong AH, Leung ST, Chin TW, Lo CSY, et al. Frequency and distribution of chest radiographic findings in COVID-19 positive patients. Radiology. 2020 Aug;296(2):E72-8.

9 Wang Y, Dong C, Hu Y, Li C, Ren Q, Zhang $\mathrm{X}$, et al. Temporal changes of CT findings in 90 patients with COVID-19 pneumonia: a Longitudinal Study. Radiology. 2020 Aug; 296:E55-64.

10 Pan F, Ye T, Sun P, Gui S, Liang B, Li L, et al. Time course of lung changes at chest CT dur- ing recovery from coronavirus disease 2019 (COVID-19). Radiology. 2020 Jun;295:71521.

11 Daoussis D, Leonidou L, Kalogeropoulou C, Paliogianni F, Tzouvelekis A. Protracted severe COVID-19 pneumonia following rituximab treatment: caution needed. Rheumatol Int. 2021 Oct;41(10):1839-43.

12 Riches JC. Impact of COVID-19 in patients with lymphoid malignancies. World J Virol. 2021 May 25;10(3):97-110.

13 Reuken PA, Stallmach A, Pletz MW, Brandt C, Andreas N, Hahnfeld S, et al. Severe clinical relapse in an immunocompromised host with persistent SARS-CoV-2 infection. Leukemia. 2021 Mar;35:920-3.

14 Yasuda H, Mori Y, Chiba A, Bai J, Murayama G, Matsushita Y, et al. Resolution of one-year persisting COVID-19 pneumonia and development of immune thrombocytopenia in a follicular lymphoma patient with preceding rituximab maintenance therapy: a follow-up report and literature review of cases with prolonged infections. Clin Lymphoma Myeloma Leuk. 2021 Oct;21:e810-6.

15 Raoufi M, Kahkooei S, Haseli S, Robatjazi F, Bahri J, Khalili N. Organizing pneumonialike pattern in COVID-19. Adv Respir Med. 2021 Oct 6. Epub ahead of print.

16 Garrana SH, Som A, Ndakwah GS, Yeung T, Febbo J, Heeger AP, et al. Comparison of chest CT Findings of COVID-19, influenza, and organizing pneumonia: a Multireader Study. AJR Am J Roentgenol. 2021 Nov; 217(5):1093-102.

17 Cheson BD, Fisher RI, Barrington SF, Cavalli F, Schwartz LH, Zucca E, et al. Recommendations for initial evaluation, staging, and response assessment of Hodgkin and nonHodgkin lymphoma: the Lugano classification. J Clin Oncol. 2014 Sep 20;32:3059-68.

18 Zamagni E, Tacchetti P, Cavo M. Imaging in multiple myeloma: how? When? Blood. 2019 Feb 14;133:644-51.

19 Katal S, Azam S, Bombardieri E, Picchio M, Gholamrezanezhad A. Reopening the country: recommendations for nuclear medicine departments. World J Nucl Med. 2021 JanMar;20(1):1-6.

20 Sreedharan S, Mian M, McArdle DJT, Rhodes A. The impact of the COVID-19 pandemic on diagnostic imaging services in Australia. J Med Imaging Radiat Oncol. 2021 Jul 20:10. Epub ahead of print.

21 Norbash AM, Moore AV, Recht MP, Brink JA, Hess CP, Won JJ, et al. Early-stage radiology volume effects and considerations with the coronavirus disease 2019 (COVID-19) pandemic: adaptations, risks, and lessons learned. J Am Coll Radiol. 2020 Sep;17:108695.

22 Joob B, Wiwanitkit V. 18F-FDG PET/CT and COVID-19. Eur J Nucl Med Mol Imaging. 2020 Jun;47:1348.

23 Guidance and best practices for nuclear cardiology laboratories during the coronavirus disease 2019 (COVID-19) pandemic: an Information Statement from ASNC and SNMMI. J Nucl Med 2020 Jun;61:784-91.

24 Gnanasegaran G, Huang HL, Williams J, Bomanji J. Coronavirus pandemic: what nuclear medicine departments should know. J Nucl Med Technol. 2020 Jun;48:89-97.

25 Qin C, Liu F, Yen TC, Lan X. 18F-FDG PET/ CT findings of COVID-19: a series of four highly suspected cases. Eur J Nucl Med Mol Imaging. 2020 May;47:1281-6.

26 Deng Y, Lei L, Chen Y, Zhang W. The potential added value of FDG PET/CT for COVID-19 pneumonia. Eur J Nucl Med Mol Imaging. 2020 Jul;47:1634-5.

27 Charters PFP, Little D, Rodrigues JCL, Graham RN, Redman SL. 18FDG-PET/CT findings in COVID-19: a single centre retrospective radiological review. BJR Case Rep. 2020 Sep 1;6:20200091.

28 Minamimoto R, Hotta M, Ishikane M, Inagaki T. FDG-PET/CT images of COVID-19: a comprehensive review. Glob Health Med. 2020 Aug 31;2(4):221-6

29 Colandrea M, Gilardi L, Travaini LL, Fracassi SLV, Funicelli L, Grana CM. 18F-FDG PET/ $\mathrm{CT}$ in asymptomatic patients with $\mathrm{CO}$ VID-19: the submerged iceberg surfaces. Jpn J Radiol. 2020 Nov;38:1007-11.

30 Zou S, Zhu X. FDG PET/CT of COVID-19. Radiology. 2020 Aug;296:E118.

31 Amini H, Divband G, Montahaei Z, Dehghani T, Kaviani H, Adinehpour Z, et al. A case of COVID-19 lung infection first detected by [18F]FDG PET-CT. Eur J Nucl Med Mol Imaging. 2020 Jul;47:1771-2. 
32 Kamani $\mathrm{CH}$, Jreige M, Pappon M, Fischbacher A, Borens O, Monney P, et al. Added value of $18 \mathrm{~F}-\mathrm{FDG}$ PET/CT in a SARS-CoV-2-infected complex case with persistent fever. Eur J Nucl Med Mol Imaging. 2020 Jul;47:2036-7.

$33 \mathrm{Xu}$ X, Yu C, Qu J, Zhang L, Jiang S, Huang D, et al. Imaging and clinical features of patients with 2019 novel coronavirus SARS-CoV-2. Eur J Nucl Med Mol Imaging. 2020 May;47: 1275-80.

34 Karimi-Galougahi M, Yousefi-Koma A, Bakhshayeshkaram M, Raad N, Haseli S. 18FDG PET/CT scan reveals hypoactive orbitofrontal cortex in anosmia of COVID-19. Acad Radiol. 2020 Jul;27:1042-3.

35 Halsey R, Priftakis D, Mackenzie S, Wan S, Davis LM, Lilburn D, et al. COVID-19 in the act: incidental 18F-FDG PET/CT findings in asymptomatic patients and those with symptoms not primarily correlated with COVID-19 during the United Kingdom coronavirus lockdown. Eur J Nucl Med Mol Imaging. 2021 Jan;48:269-81.

36 Fang Y, Zhang H, Xie J, Lin M, Ying L, Pang $\mathrm{P}$, et al. Sensitivity of chest CT for COVID-19: comparison to RT-PCR. Radiology. 2020 Aug;296:E115-7.

37 Meena JP, Kumar Gupta A, Tanwar P, Ram Jat K, Mohan Pandey R, Seth R. Clinical presentations and outcomes of children with cancer and COVID-19: a systematic review. Pediatr Blood Cancer. 2021 Jun;68(6):e29005.

38 Karasu S, Sinci KA, Yildiz Y, Gumus C, Tosun O. Chemotherapy-induced pulmonary toxicity mimicking COVID-19 pneumonia. Curr Med Imaging. 2021;17(12):1510-12.

39 Dai Y, Liu S, Zhang Y, Li X, Zhao Z, Liu P, et al. A false alarm of COVID-19 pneumonia in lung cancer with anti-PD-1 related pneumonitis: a case report and review of the literature. J Med Case Rep. 2021 Feb 1;15:41.

40 Rossi E, Schinzari G, Tortora G. Pneumonitis from immune checkpoint inhibitors and $\mathrm{CO}$ VID-19: current concern in cancer treatment. J Immunother Cancer. 2020 Jul;8(2):e000952.

41 Prakash S, Kumar R, Vinjamuri S. What role does 18F-fluorodeoxyglucose PET-computed tomography have in the current pandemic and beyond? Nucl Med Commun. 2021 Feb; 42(2):113-6.

42 Giannakis A, Móré D, Erdmann S, Kintzelé L, Fischer RM, Vogel MN, et al. COVID-19 pneumonia and its lookalikes: how radiologists perform in differentiating atypical pneumonias. Eur J Radiol. 2021 Oct 19;144:110002.
43 Tahtabasi M, Hosbul T, Karaman E, Akin Y, Konukoglu O, Sahiner F. Does COVID-19 cause an increase in spleen dimensions? Possible effects of immune activation, hematopoietic suppression and microthrombosis. Clin Imaging. 2021 Nov;79:104-9.

44 Ramanathan M, Chueng T, Fernandez E, Gonzales-Zamora J. Concomitant renal and splenic infarction as a complication of COVID-19: a case report and literature review. Infez Med. 2020 Dec 1;28:611-5.

45 Vaidya T, Nanivadekar A, Patel R. Imaging spectrum of abdominal manifestations of COVID-19. World J Radiol. 2021 Jun 28; 13(6):157-70.

46 Ghalib N, Pophali P, Chamorro-Pareja N, Jayarangaiah A, Kumar A. Incidental asymptomatic splenic infarct in a COVID-19 patient. Cureus. 2021 Feb 1;13(2):e13065.

47 de Roquetaillade C, Chousterman BG, Tomasoni D, Zeitouni M, Houdart E, Guedon A, et al. Unusual arterial thrombotic events in Covid-19 patients. Int J Cardiol. 2021 Jan 15;323: 281-4.

48 Tsaras G, Owusu-Ansah A, Boateng FO, Amoateng-Adjepong Y. Complications associated with sickle cell trait: a brief narrative review. Am J Med. 2009 Jun;122:507-12.

49 Yasmin F, Najeeb H, Naeem A, Dapke K, Phadke R, Asghar MS, et al. COVID-19 associated mucormycosis: a systematic review from diagnostic challenges to management. Diseases. 2021 Sep 22;9(4):65.

50 Singh AK, Singh R, Joshi SR, Misra A. Mucormycosis in COVID-19: a systematic review of cases reported worldwide and in India. Diabetes Metab Syndr. 2021 Jul-Aug; 15(4): 102146.

51 Maini A, Tomar G, Khanna D, Kini Y, Mehta $\mathrm{H}$, Bhagyasree V. Sino-orbital mucormycosis in a COVID-19 patient: a case report. Int J Surg Case Rep. 2021 May 4;82:105957.

52 Dimopoulos G, Almyroudi MP, Myrianthefs P, Rello J. COVID-19-associated pulmonary aspergillosis (CAPA). J Intensive Med. 2021 Aug; 1(2):71-80.

53 Ufuk F, Bulgurcu E, Sari T. COVID-19-associated pancytopenia and typhlitis. Am J Emerg Med. 2021 Jan 4;45:685.e1-e3.

54 Eifer M, Eshet Y. Imaging of COVID-19 vaccination at FDG PET/CT. Radiology. 2021 May;299(2):E248.

55 Shirone N, Shinkai T, Yamane T, Uto F, Yoshimura $\mathrm{H}$, Tamai $\mathrm{H}$, et al. Axillary lymph node accumulation on FDG-PET/CT after influenza vaccination. Ann Nucl Med. 2012 Apr;26:248-52.
56 Burger IA, Husmann L, Hany TF, Schmid DT, Schaefer NG. Incidence and intensity of F-18 FDG uptake after vaccination with H1N1 vaccine. Clin Nucl Med. 2011 Oct;36: 848-53.

57 Thomassen A, Lerberg Nielsen A, Gerke O, Johansen A, Petersen H. Duration of 18FFDG avidity in lymph nodes after pandemic H1N1v and seasonal influenza vaccination. Eur J Nucl Med Mol Imaging. 2011 May;38: 894-8.

58 Bernstine $\mathrm{H}$, Priss $\mathrm{M}$, Anati T, Turko O, Gorenberg M, Steinmetz AP, et al. Axillary lymph nodes hypermetabolism after BNT162b2 mRNA COVID-19 vaccination in cancer patients undergoing 18F-FDG PET/ CT: a Cohort Study. Clin Nucl Med. 2021 May 1;46:396-401.

59 Cohen D, Krauthammer SH, Wolf I, EvenSapir E. Hypermetabolic lymphadenopathy following administration of BNT162b2 mRNA Covid-19 vaccine: incidence assessed by [18F]FDG PET-CT and relevance to study interpretation. Eur J Nucl Med Mol Imaging. 2021 Jun;48(6):1854-63.

60 Eifer M, Tau N, Alhoubani Y, Kanana N, Domachevsky L, Shams J, et al. Covid-19 mRNA vaccination: age and immune status and its association with axillary lymph node PET/CT uptake. J Nucl Med. 2022 Jan;63(1): 134-9.

61 Orevi M, Chicheportiche A, Ben-Haim S. Lessons learned from post-COVID-19 vaccination PET/CT studies. J Nucl Med. 2021 Jul 22: jnumed. Epub ahead of print.

62 Eshet Y, Tau N, Alhoubani Y, Kanana N, Domachevsky L, Eifer M. Prevalence of increased FDG PET/CT axillary lymph node uptake beyond 6 weeks after mRNA COVID-19 vaccination. Radiology. 2021 Sep; 300(3):E345-7.

63 Klok FA, Pai M, Huisman MV, Makris M. Vaccine-induced immune thrombotic thrombocytopenia. Lancet Haematol. 2022; 9(1):E78-80.

64 Perry RJ, Tamborska A, Singh B, Craven B, Marigold R, Arthur-Farraj P, et al. Cerebral venous thrombosis after vaccination against COVID-19 in the UK: a multicentre cohort study. Lancet. 2021 Sep 25;398:1147-56.

65 Greinacher A, Selleng K, Palankar R, Wesche J, Handtke S, Wolff $\mathrm{M}$, et al. Insights in ChAdOx1 nCoV-19 vaccine-induced immune thrombotic thrombocytopenia. Blood. 2021 Dec 2;138(22):2256-68. 